\title{
Soluble Triggering Receptors Expressed on Myeloid Cells-I as a Neonatal Ventilator-Associated Pneumonia Biomarker
}

\author{
Zu-Qin Yang' \\ Jing-Yun $\mathrm{Mai}^{2}$ \\ Min-Li Zhu ${ }^{2}$ \\ Xiu-Man Xiao ${ }^{2}$ \\ Xiao-Xiao $\mathrm{He}^{2}$ \\ Shang-Qin Chen ${ }^{2}$ \\ Zhen-Lang Lin $^{2}$ \\ Xing Feng'
}

'Department of Neonatology, Children's Hospital of Soochow University, Suzhou, 215025, Jiangsu, People's Republic of China; ${ }^{2}$ Department of Neonatology, The Second Affiliated Hospital and Yuying Children's Hospital of Wenzhou Medical University, Wenzhou, 325027, People's

Republic of China
Correspondence: Xing Feng Department of Neonatology, Children's Hospital of Soochow University, No. 92 Zhongnan Street, Wuzhong District, Suzhou, 2 I5025, Jiangsu, People's Republic of China

Tel +86051280692700

Fax +86051280693599

Email fengxing1938@2Icn.com
Background: Neonatal ventilator-associated pneumonia (NVAP) is one of the main infections acquired in hospitals, and soluble triggering receptors expressed on myeloid cells-1 (sTREM-1) are a TREM-1 subtype that can be released into the blood or bodily fluids during an infection.

Methods: The patients included in the present study were divided into three groups: the NVAP group, the first control group, and the second control group ( $\mathrm{n}=20$, each). Children requiring respiratory treatment were assigned to the NVAP group, newborns who received mechanical ventilation and had neonatal respiratory distress syndrome were assigned to the first control group, and newborns with normal X-ray and electrocardiogram results but no non-pulmonary infection was assigned to the second control group. The blood and bronchoalveolar lavage fluid (BALF) sTREM-1 levels in all newborns were analyzed.

Results: The acute-phase blood and BALF sTREM-1 levels were significantly higher in the NVAP group than in the first control group, and the blood sTREM-1 expression level was lower in the second control group than in the NVAP group.

Conclusion: The present results suggest that sTREM-1 might be a useful biomarker for NVAP prediction in the Department of Pediatrics.

Keywords: pneumonia, neonatal ventilator-associated pneumonia, soluble TREM-1, neonate, C-reactive protein

\section{Background}

Neonatal ventilator-associated pneumonia (NVAP) is a common nosocomial infection as well as one of the main causes of mechanical ventilation failure. It is defined as a lower airway infection in intubated patients with an onset after $\geq 48 \mathrm{~h}$ of invasive mechanical ventilation. ${ }^{1}$ The NVAP infection rate in newborns can reach up to $6.8-32.2 \% .^{2}$ Furthermore, in child patients, NVAP occurrence prolongs inpatient time and increases mortality and inpatient costs; this may lead to the generation of multiple-resistant strain. ${ }^{3}$

Triggering receptors expressed on myeloid cells-1 (TREM-1) are an important member of the immunoglobulin superfamily; they play the role of a positive regulator in the immune response following microorganism risk factor identification. Soluble TREM-1 (sTREM-1) is a TREM-1 subtype that can be released into the blood or bodily fluids during an infection. ${ }^{4}$ The sTREM-1 level has been used as a marker for identifying infection and assessing inflammation severity in adult patients with septicemia, ventilator-associated pneumonia (VAP), and severe 
pancreatitis. Chao et $\mathrm{al}^{5}$ found that the $\mathrm{C}$-reactive protein (CRP) and sTREM-1 can be used to forecast noncommunity-acquired pneumonia (non-CAP) in adult patients on the third day. The different sTREM-1 and CRP trends in patients with non-CAP indicate that STREM-1 is a kind of auxiliary forecasting biomarker in patients with CRP-relative CAP. Zhong et $\mathrm{al}^{6}$ found that among 60 child patients with pneumonia, 25 had bacterial pneumonia, 17 had viral pneumonia, and 18 had mycoplasma pneumonia. Furthermore, CD64 and sTREM-1 expressions were obviously higher among patients with mycoplasma pneumonia than in patients with viral pneumonia, patients with mycoplasma pneumonia, and patients in the study's control groups $(p<0.05)$. Li et al ${ }^{7}$ suggested that the detection of sTREM-1 in bronchoalveolar lavage fluid (BALF) and exhaled ventilator condensate samples may be useful for VAP diagnosis in children after heart surgery. Yu et $\mathrm{al}^{8}$ reported that sTREM-1 in the BALF and exhaled ventilator condensate had good diagnostic performances in differentiating between VAP-positive and VAPnegative patients with ischemia. However, Palazzo et al showed that sTREM-1 failed to categorize adult patients as VAP-positive or VAP-negative. ${ }^{9}$ Few studies on the correlation between sTREM-1 and NVAP have been conducted; therefore, the present study aimed to investigate the predictive performance of sTREM-1 in NVAP.

\section{Methods}

\section{Patients}

The patients included in the present study were divided into three groups: the NVAP group, the first control group, and the second control group $(n=20$, each). Child patients in the Department of Neonatology of the Second Affiliated Hospital, Wenzhou Medical University between April 2014 and April 2016 who required respiratory treatment and were diagnosed with NVAP were assigned to the NVAP group; newborns who received mechanical ventilation and had neonatal respiratory distress syndrome were assigned to the first control group; and newborns with normal X-ray and electrocardiogram results who presented with non-pulmonary infection, such as hyperbilirubinemia, hypoxic-ischemic encephalopathy, hypoglycemia, and hypocalcemia, were assigned to the second control group. The male-to-female ratio was 1:1 in all groups. The exclusion criteria for all groups were: (1) patients with an incomplete medical record; (2) patients with severe systemic infection; and (3) patients who underwent mechanical ventilation for $<5$ days.

BALF was collected from the tracheal intubation for newborns on the day of diagnosis after the bronchus and flushed with $3 \mathrm{~mL}$ sterile purified water. The present study gained the agreement and was approved by the ethics committee of the Second Affiliated Hospital, Wenzhou Medical University.

\section{NVAP Diagnostic Criteria}

NVAP was diagnosed according to the following criteria: (1) fever or increased body temperature; (2) purulent sputum in the trachea and bronchus as well as visible bacteria on the smear stain; (3) new or progressive lung infiltrations on the chest X-ray; and (4) a positive trachea secretion culture and a colony count of $>10^{6} / \mathrm{mL}$. A low-power microscope with $>25$ white blood cells (WBCs) and $<10$ squamous cells was used for sputum culture.

\section{Blood and BALF Sample Collection}

The blood and BALF samples were collected from all subjects immediately after the recruitment (during the acute phase). A total of $2 \mathrm{~mL}$ venous blood was collected from all subjects, 10\% EDTA was used for anticoagulation, and centrifugation (2000 rpm) was conducted for 30 minutes to separate the plasma. Next, the liquid supernatant was transferred into a sterile $1 \mathrm{~mL}$ EP tube. The tube was then labelled, tightly covered, and placed into a refrigerator at $-70^{\circ} \mathrm{C}$ for cryopreservation and subsequent one-time detection. BALF preparation and collection as well as fiberoptic bronchoscope examination were conducted, as previously described. ${ }^{10}$ The samples were filtered using single-layer gauze for viscous liquid removal and placed into a refrigerator at $4{ }^{\circ} \mathrm{C}$ for storage. Centrifugation (3000 rpm, 10 minutes) was then carried out within three hours, and the liquid supernatant was removed and placed into a disinfected $1000-\mu \mathrm{L}$ EP tube. All samples were placed in a refrigerator at $-70^{\circ} \mathrm{C}$ for cryopreservation and subsequent one-time detection.

\section{sTREM-I Detection}

A double-antibody enzyme-linked immunosorbent assay kit (\#HL-192018H, Haling Biotechnology, Shanghai, China) was used to detect plasma and BALF sample sTREM-1 expression levels in accordance with the manufacturer's instructions. The assay was performed in duplicate. The sTREM-1 level was normalized by the total protein concentration. A Multiskan MS Type 352 
(Labsystems, Finland) automated microplate reader was used for detection, and the absorbance (optical density [OD] value) was read at a wavelength of $450 \mathrm{~nm}$. Next, a standard curve was drawn to read the sample detection concentration, with the OD value as the vertical coordinate. The blood and BALF sTREM-1 concentrations before and after treatment were compared between the NVAP group and the first control group, and the detected blood sTREM-1 concentration values were compared among the three groups. Lower respiratory tract detection and bacterial culture were then performed in the NVAP group and the first control group.

\section{Statistical Analysis}

The data analysis was conducted using the Statistical Package for Social Sciences 18.0 software, and the normality of data was assessed using the KolmogorovSmirnov test. The measurement results were presented as mean \pm standard deviation $(\bar{x} \pm \mathrm{SD})$. Comparisons between two groups were verified with two separate samples using the $t$-test method. A $\mathrm{p}$ value of $<0.05$ was considered statistically significant in the determination of the plasma and BALF sTREM-1 concentration NVAP diagnostic value.

\section{Results}

\section{Basic Neonate Characteristics (All Groups)}

The average birth weight was $2.01 \pm 1.12 \mathrm{~kg}$ in the NVAP group, $2.13 \pm 0.98 \mathrm{~kg}$ in the first control group, and $2.21 \pm$ $1.01 \mathrm{~kg}$ in the second control group. The average gestational age was $29.8 \pm 4.7$ weeks (25-39 weeks) in the first control group, and $34.7 \pm 2.4$ weeks (33-42 weeks) in the second control group; the NVAP group had a gestational age of 25-40 weeks, with an average gestational age of $29.3 \pm 5.1$ weeks. The number of caesarean section cases was 11 in the NVAP group, 9 in the first control group, and 12 in the second control group. There were no significant differences in the above-mentioned characteristics among the three groups; however, there were significant differences in the numbers of premature among the three groups (NVAP group: 16; first control group: 13; and second control group: 6) (Table 1).

\section{Group Acute-Phase Serum and BALF sTREM-I Measurement}

The BALF sample sTREM-1 values were $373.39 \pm 29.76$ $\mathrm{pg} / \mathrm{mL}$ in the NVAP group and $331.13 \pm 22.57 \mathrm{pg} / \mathrm{mL}$ in the first control group; the average sTREM-1 level was $306.26 \pm 34.98 \mathrm{pg} / \mathrm{mL}$ in the NVAP group and $273.94 \pm$ $15.67 \mathrm{pg} / \mathrm{mL}$ in the first control group; and the serum sTREM-1 level in the second control group was 270.88 \pm 22.09 . No sTREM-1 was detected in the second control group BALF samples.

The statistical analysis of serum sTREM-1 revealed significant differences when comparing the NVAP group with the first control group $(p<0.001)$. However, there were no significant differences in blood sTREM-1 when comparing the first control group with the second control group ( $p=0.415)$. Furthermore, there was a significant difference in blood sTREM-1 when comparing the NVAP group with the second control group $(p=0.001)$. Hence, it can be observed that acute-phase blood and BALF sTREM-1 expressions were relatively high in the NVAP group. In contrast, the blood sTREM-1 level was lowly expressed in first control group and the second control group; the difference was not statistically significant (Figure 1).

\section{BALF sTREM-I Measurement in Patients with NVAP and Bacterial-Culture-Positive Serum}

The average BALF sTREM1 value was $387.59 \pm 38.95$ $\mathrm{pg} / \mathrm{mL}$ in gram-negative $\left(\mathrm{G}^{-}\right)$bacteria-infected patients and $363.92 \pm 17.90 \mathrm{pg} / \mathrm{mL}$ in non-G- bacteria-infected patients (including fungi and gram-positive $[\mathrm{G}+]$ bacteria);

Table I Basic Characteristics of Enrolled Neonates

\begin{tabular}{|c|c|c|c|c|}
\hline & Control I $(n=20)$ & Control $2(n=20)$ & NVAP $(n=20)$ & \\
\hline Male/Female & $\mathrm{I}: \mathrm{I}$ & $1: 1$ & I:I & \\
\hline Birth weight (kg) & $2.13 \pm 0.98$ & $2.21 \pm 1.01$ & $2.01 \pm 1.12$ & $p>0.05$ \\
\hline Gestational age (weeks) & $29.8 \pm 4.7$ & $34.7 \pm 2.4$ & $29.3 \pm 5.1$ & $p>0.05$ \\
\hline Number of Caesarean section & $9(45 \%)$ & $12(60 \%)$ & II (55\%) & $p>0.05$ \\
\hline Number of premature & $13(65 \%)$ & $6(30 \%)$ & $16(80 \%)$ & $p<0.05$ \\
\hline
\end{tabular}




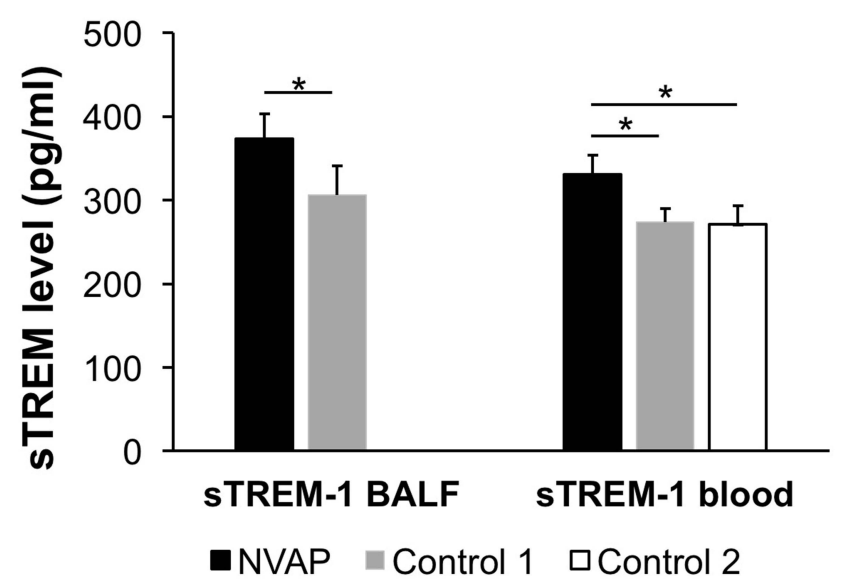

Figure I Acute-phase sTREM-I comparison between the NVAP group and the control groups. The sTREM-I levels in the NVAP, first control group, and second control group are shown. No sTREM-I was detected in the second control group BALF samples. ${ }^{*} \mathrm{p}<0.05$.

the difference was not statistically significant $(\mathrm{p}=0.110)$. Furthermore, the blood sTREM-1 value was $341.39 \pm$ $17.19 \mathrm{pg} / \mathrm{mL}$ in $\mathrm{G}-$ bacteria-infected child patients and $324.28 \pm 23.76 \mathrm{pg} / \mathrm{mL}$ in non-G- bacteria-infected child patients; the difference was not statistically significant $(\mathrm{p}=$ 0.190). The NVAP group blood and BALF sample sTREM-1 values are shown in Supplementary Table 1.

\section{Discussion}

TREM-1 are mainly expressed in neutrophile granulocyte, mature monocyte, and the surface of macrophages. ${ }^{11}$ The cascade reaction induces the production of proinflammatory cytokines, such as tumor necrosis factor alpha, monocyte chemoattractant protein 1, and interleukin-1 $\beta$, after bacterial infection. ${ }^{12}$ Furthermore, TREM-1 can affect the pathological process of septic shock. ${ }^{13}$ Hence, it plays an important role in the inflammatory reaction triggering and amplification process. ${ }^{14,15}$ sTREM-1 are the acute phase product of bacterial infection. ${ }^{16}$ Detection of sTREM-1 in the bodily fluids of a living body has a great application value for bacterial infection diagnosis. ${ }^{17,18}$ According to a previous report, ${ }^{19}$ the sTREM-1 level was increased in BALF obtained from patients with pneumonia and septicemia, plasma obtained from newborn patients with septicemia, and the exhaled breath condensate of patients with NVAP. In addition, detection of sTREM-1 in sputum, pleural effusion, cerebrospinal fluid, urine, and arthritis also has an important clinical value for the identification of relevant infectious diseases. $^{20}$
However, sTREM-1 level detection cannot quickly identify systemic inflammatory response syndrome (SIRS) and septicemia or delay the occurrence of septicemia in newborns; ${ }^{21}$ however, it can be used to reflect septicemia severity. Dynamic serum sTREM-1 monitoring helps physicians determine child patient prognoses. $^{22}$ Therefore, serum sTREM-1 was considered capable of effectively identifying septicemia and SIRS. With a plasma sTREM-1 level of $60 \mathrm{ng} / \mathrm{mL}$ as the cut-off value, the sensitivity and specificity for septicemia diagnosis are $96 \%$ and $89 \%$, respectively. Giamarellos-Bourboulis et $\mathrm{al}^{23}$ studied patients with VAP-induced septicemia and found that the plasma sTREM-1 level was continuously higher in the death group than in the survival group. Zhang et $\mathrm{al}^{24}$ studied 52 patients with septicemia and found that the serum sTREM-1 level was higher in patients with severe septicemia than in patients with normal septicemia during the first day of hospitalization $(240.6 \mathrm{pg} / \mathrm{mL}$ vs $118.3 \mathrm{pg} / \mathrm{mL}$; $\mathrm{p}<0.01)$. The serum sTREM-1 level gradually decreased in the survival group and gradually increased in the death group. The serum sTREM-1 level was lower in the survival group than in the death group at all time points. Matsuno $\mathrm{AK}_{\text {et }} \mathrm{al}^{25}$ also found detecting EVC sTREM-1 to be one of the most important diagnostic approaches in VAP after heart surgery. The TREM-1 expression was higher in child patients with sepsis than in healthy children and higher in child patients with severe sepsis than in patients with general sepsis. ${ }^{26}$ Alkan Ozdemir et $\mathrm{al}^{27}$ found that sTREM-1 expression in newborns was obviously higher in the culture verification group than in the suspected sepsis group. Urine sTREM-1 might be a reliable and sensitive marker for detecting sepsis in premature infants. Adly et al ${ }^{10}$ assessed 112 newborns with septicemia (63 positive cultures, 49 negative cultures, and 40 healthy newborns) and revealed that sTREM-1 was obviously higher in newborns with early sepsis than in newborns with late sepsis, and that it was related to mortality. The sTREM-1 was obviously reduced after antibiotic treatment for 48 hours compared with the samples obtained from continuously positive-cultured newborns. Furthermore, sTREM-1 was positively correlated with the WBC count, neutrophile granulocyte absolute count, immature/total neutrophil granulocyte specific value, high sensitivity CRP (hs-CRP), and sepsis score; however, it was negatively correlated with gestational age and weight.

A meta-analysis by Bellos et al suggested that sTREM1 could be used as a useful biomarker for neonatal sepsis 
prediction. ${ }^{28}$ However, few correlation studies on STREM1 in NVAP have been conducted, and there are presently no related reports on NVAP in China. The present study revealed that the serum STREM-1 level was significantly higher in the NVAP group than in both control groups and that the BALF sTREM-1 expression was evidently higher in the NVAP group than in first control group. Hence, the sTREM-1 level exhibits a parallel dynamic change with the illness state.

The results of the present study further showed no significant difference between the blood and BALF sample sTREM-1 value in $\mathrm{G}^{-}$- bacteria-infected child patients and non-G- bacteria-infected child patients. Bouchon et $\mathrm{al}^{3}$ found that the $\mathrm{G}^{-}$- bacteria-generated lipopolysaccharide significantly improved sTREM-1 expression when compared with other bacteria. The present study failed to determine whether there were obvious differences between the sTREM-1 level in $\mathrm{G}-$ bacteria and $\mathrm{G}+$ bacteria. However, the average $\mathrm{G}^{-}$- detection value was basically $>\mathrm{G}+$; this may be correlated with the state of child patients with clinically severe $\mathrm{G}^{-}$infection illness and the prognosis being poor.

The laboratory bacterial infection indexes, such as the WBC count, neutrophil granulocyte ratio, CRP and PCT, ${ }^{29}$ and pancreatic stone protein, ${ }^{30}$ cannot satisfy clinical demands. Bacterial culture during the NVAP diagnosis is the gold standard; however, the analysis speed is low, and the method has the limitation of a false negative result. Furthermore, the physiological range of newborns has a relatively large fluctuation, and when an organism with low immunization or hepatic failure becomes seriously infected, WBC, PCT, and CRP sensitivity and specificity are greatly affected. Other acute-phase proteins (serum amyloid protein A, procalcitonin, lipopolysaccharide binding protein, and mannan-binding lectin) have an intermediate diagnostic accuracy. ${ }^{31}$

There are several limitations to the present study: (1) follow-up data were not obtained from the patients, and further analysis is required to evaluate the long-term $(\geq$ one month) prognostic value of sTREM1 as a biomarker for NVAP outcome prediction; and (2) further larger size studies are required to determine the optimal sTREM1 cutoff value for NVAP diagnosis.

\section{Conclusions}

The results of the present study demonstrate that the serum and BALF sTREM-1 levels were significantly higher in patients with NVAP when compared with the control subjects. These findings suggest that sTREM-1 may be a useful biomarker for NVAP prediction.

\section{Ethics Approval and Consent to Participate}

This study was conducted in accordance with the declaration of Helsinki. This study was conducted with approval from the Ethics Committee of the Second Affiliated Hospital, Wenzhou Medical University. A written informed consent was obtained from all participants.

\section{Funding}

Wen zhou Science and Technology Plan Project (2017Y0622).

\section{Disclosure}

The authors report no conflicts of interest in this work.

\section{References}

1. Goerens A, Lehnick D, Büttcher M, et al. Neonatal ventilator associated pneumonia: a quality improvement initiative focusing on antimicrobial stewardship. Front Pediatr. 2018;6:262. doi:10.3389/fped.2018.00262

2. Azab SF, Sherbiny HS, Saleh SH, et al. Reducing ventilatorassociated pneumonia in neonatal intensive care unit using "VAP prevention bundle": a cohort study. BMC Infect Dis. 2015;15:314. doi:10.1186/s12879-015-1062-1

3. Bouchon A, Dietrich J, Colonna M. Cutting edge: inflammatory responses can be triggered by TREM-1, a novel receptor expressed on neutrophils and monocytes. J Immunol. 2000;164(10):4991-4995. doi:10.4049/jimmunol.164.10.4991

4. Su L, Feng L, Song Q, et al. Diagnostic value of dynamics serum sCD163, sTREM-1, PCT, and CRP in differentiating sepsis, severity assessment, and prognostic prediction. Mediators Inflamm. 2013;2013:969875. doi:10.1155/2013/969875

5. Chao WC, Wang CH, Chan MC, Chow KC, Hsu JY, Wu CL. Predictive value of serial measurements of sTREM-1 in the treatment response of patients with community-acquired pneumonia. J Formos Med Assoc. 2007;106(3):187-195. doi:10.1016/S0929-6646(09)60239-4

6. Zhong MF, Zhao JM. Diagnostic values of plasma CD64 and sTREM-1 for pediatric pneumonia. Zhongguo Dang Dai Er Ke Za Zhi. 2016;18(7):599-602.

7. Li C, Zhu L, Gong X, et al. Soluble triggering receptor expressed on myeloid cells-1 as a useful biomarker for diagnosing ventilator-associated pneumonia after congenital cardiac surgery in children. Exp Ther Med. 2019;17(1):147-152.

8. Yu Y, Zhu C, Liu C, Gao Y, Yin R, Cao J. Diagnostic performance of soluble triggering receptor expressed on myeloid cells-1 in ventilator-associated pneumonia of patients with ischemic stroke. Can J Infect Dis Med Microbiol. 2017;2017:9513690. doi:10.1155/2017/ 9513690

9. Palazzo SJ, Simpson TA, Simmons JM, Schnapp LM. Soluble triggering receptor expressed on myeloid cells-1 (sTREM-1) as a diagnostic marker of ventilator-associated pneumonia. Respir Care. 2012;57(12):2052-2058. doi:10.4187/respcare.01703

10. Adly AA, Ismail EA, Andrawes NG, El-Saadany MA. Circulating soluble triggering receptor expressed on myeloid cells-1 (sTREM-1) as diagnostic and prognostic marker in neonatal sepsis. Cytokine. 2014;65(2):184-191. doi:10.1016/j.cyto.2013.11.004 
11. Zysset D, Weber B, Rihs S, et al. TREM-1 links dyslipidemia to inflammation and lipid deposition in atherosclerosis. Nat Commun. 2016;7:13151. doi:10.1038/ncomms13151

12. Kany S, Vollrath JT, Relja B. Cytokines in inflammatory disease. Int J Mol Sci. 2019;20(23):6008. doi:10.3390/ijms20236008

13. Qian L, Weng XW, Chen W, Sun CH, Wu J. TREM-1 as a potential therapeutic target in neonatal sepsis. Int J Clin Exp Med. 2014;7 (7):1650-1658.

14. Nathan C, Ding A. TREM-1: a new regulator of innate immunity in sepsis syndrome. Nat Med. 2001;7(5):530-532. doi:10.1038/87846

15. Yang C, Chen B, Zhao J, et al. TREM-1 signaling promotes host defense during the early stage of infection with highly pathogenic streptococcussuis. Infect Immun. 2015;83(8):3293-3301. doi:10.1 128/IAI.00440-15

16. Su L, Liu D, Chai W, Liu D, Long Y. Role of sTREM-1 in predicting mortality of infection: a systematic review and meta-analysis. $B M J$ Open. 2016;6(5):e010314. doi:10.1136/bmjopen-2015-010314

17. Ruiz-González A, Esquerda A, Falguera M, et al. Triggering receptor (TREM-1) expressed on myeloid cells predicts bacteremia better than cliniacal variables in community-acquired penuminia. Respirlogy. 2011;16(2):321-325. doi:10.1111/j.1440-1843.2010.01905.x

18. Liu XR, Xu J, Wang YM, Ji MS, Liu FS. The effects of paeoniflorin injection on soluble triggering receptor expressed on myeloid-1 (sTREM-1) levels in severe septic rats. Korean $J$ Physiol Pharmacol. 2016;20(6):565-571. doi:10.4196/kjpp.2016.20.6.565

19. Phua J, Koay ES, Zhang D, et al. Soluble triggering receptor expressed on myeloid cells- 1 in acute respiratory infections. Eur Respirat J. 2006;28(4):695-702. doi:10.1183/09031936.06.00005606

20. Lemarié J, Barraud D, Gibot S. Host response biomarkers in sepsis: overview on sTREM-1 detection. Methods Mol Biol. 2015;1237:225-239.

21. Arízaga-Ballesteros V, Alcorta-García MR, Lázaro-Martínez LC, et al. Can sTREM-1 predict septic shock \& death in late-onset neonatal sepsis? A pilot study. Int J Infect Dis. 2015;30:27-32. doi:10.1016/j.ijid.2014.10.013

22. Gibot S, Kolopp-Sarda MN, Béné MC, et al. Plasma level of a triggering receptor expressed on myeloid cells-1: its diagnostic accuracy in patients with suspected sepsis. Ann Intern Med. 2004;141(1):9-15. doi:10.7326/0003-4819-141-1-200407060-00009
23. Giamarellos-Bourboulis EJ, Zakynthinos S, Baziaka F, et al. Soluble triggering receptor expressed on myeloid cells-1 as an anti-inflammato-ry mediator in sepsis. Intensive Care Med. 2006;32:237-243. doi:10.1007/s00134-005-0017-1

24. Zhang J, She D, Feng D, Jia Y, Xie L. Dynamic changes of serum soluble triggering receptor expressed on myeloid cells-1(sTREM-1) reflect sepsis severity and can predict prognosis: a prospective study. BMC Infect Dis. 2011;11:53. doi:10.1186/1471-2334-11-53

25. Matsuno AK, Carlotti AP. Role of soluble triggering receptor expressed on myeloid cells-1 for daignosing ventilator-assocaited pneumonia after cardiac surgery: anpbservational study. Matsuno Carlotti BMC Cardiovasc Disord. 2013;13:107. doi:10.1186/14712261-13-107

26. Horst SA, Linnér A, Beineke A, et al. Prognotic value and therapeutic potential of TREM-1 in Streptococcus pyogenes-induced sepsis. $J$ Innate Immun. 2013;5(6):581-590. doi:10.1159/000348283

27. Alkan Ozdemir S, Ozer EA, Ilhan O, Sutcuoglu S, Tatlı M. Diagnostic value of urine soluble triggering receptor expressed on myeloid cells (sTREM-1) for late-onset neonatal sepsis in infected preterm neonates.J. Int Med Res. 2018;46(4):1606-1616. doi:10.1177/0300060517749131

28. Bellos I, Fitrou G, Daskalakis G, Thomakos N, Papantoniou N, Pergialiotis V. Soluble TREM-1 as a predictive factor of neonatal sepsis: a meta-analysis. Inflamm Res. 2018;67(7):571-578. doi:10.1007/s00011-018-1149-4

29. Schlapbach LJ, Graf R, Woerner A, et al. Pancreatic stone protein as a novel marker for neonatal sepsis. Intensive Care Med. 2013;39 (4):754-763. doi:10.1007/s00134-012-2798-3

30. Delanghe JR, Speeckaert MM. Translational research and biomarkers in neonatal sepsis. Clin Chim Acta. 2015;451(Pt A):46-64. doi:10.1016/j.cca.2015.01.031

31. Autilio C, Shankar-Aguilera S, Minucci A, Touqui L, De Luca D. Effect of cooling on lung secretory phospholipase A2 activity in vitro, ex vivo, and in vivo. Am J Physiol Lung Cell Mol Physiol. 2019;316(3):L498-L505. doi:10.1152/ajplung.00201.2018
International Journal of General Medicine

\section{Publish your work in this journal}

The International Journal of General Medicine is an international, peer-reviewed open-access journal that focuses on general and internal medicine, pathogenesis, epidemiology, diagnosis, monitoring and treatment protocols. The journal is characterized by the rapid reporting of reviews, original research and clinical studies across all disease areas. The manuscript management system is completely online and includes a very quick and fair peer-review system, which is all easy to use. Visit http://www.dovepress.com testimonials.php to read real quotes from published authors. 